\title{
Effects of Wall Shear Stress on Unsteady MHD Conjugate Flow in a Porous Medium with Ramped Wall Temperature
}

\author{
Arshad Khan ${ }^{1}$, llyas Khan ${ }^{2}$, Farhad Ali $^{1}$, Sami ulhaq ${ }^{3}$, Sharidan Shafie ${ }^{1 *}$ \\ 1 Department of Mathematical Sciences, Faculty of Science, Universiti Teknologi Malaysia, Skudai, Malaysia, 2 College of Engineering, Majmaah University, Majmaah, Saudi \\ Arabia, 3 City University of Science and Information Technology, Peshawar, Pakistan
}

\begin{abstract}
This study investigates the effects of an arbitrary wall shear stress on unsteady magnetohydrodynamic (MHD) flow of a Newtonian fluid with conjugate effects of heat and mass transfer. The fluid is considered in a porous medium over a vertical plate with ramped temperature. The influence of thermal radiation in the energy equations is also considered. The coupled partial differential equations governing the flow are solved by using the Laplace transform technique. Exact solutions for velocity and temperature in case of both ramped and constant wall temperature as well as for concentration are obtained. It is found that velocity solutions are more general and can produce a huge number of exact solutions correlative to various fluid motions. Graphical results are provided for various embedded flow parameters and discussed in details.
\end{abstract}

Citation: Khan A, Khan I, Ali F, ulhaq S, Shafie S (2014) Effects of Wall Shear Stress on Unsteady MHD Conjugate Flow in a Porous Medium with Ramped Wall Temperature. PLoS ONE 9(3): e90280. doi:10.1371/journal.pone.0090280

Editor: Christof Markus Aegerter, University of Zurich, Switzerland

Received September 11, 2013; Accepted January 29, 2014; Published March 12, 2014

Copyright: (c) 2014 Khan et al. This is an open-access article distributed under the terms of the Creative Commons Attribution License, which permits unrestricted use, distribution, and reproduction in any medium, provided the original author and source are credited.

Funding: No current external funding sources for this study.

Competing Interests: The authors have declared that no competing interests exist.

*E-mail: ridafie@yahoo.com

\section{Introduction}

In many practical situations such as condensation, evaporation and chemical reactions the heat transfer process is always accompanied by the mass transfer process. Perhaps, it is due to the fact that the study of combined heat and mass transfer is helpful in better understanding of a number of technical transfer processes. Besides, free convection flows with conjugate effects of heat and mass transfer past a vertical plate have been studied extensively in the literature due to its engineering and industrial applications in food processing and polymer production, fiber and granular insulation and geothermal systems [1-3]. Some recent attempts in this area of research are given in [4-9]. On the other hand, considerable interest has been developed in the study of interaction between magnetic field and the flow of electrically conducting fluids in a porous medium due to its applications in modern technology [10]. Toki et al. [11] have studied the unsteady free convection flows of incompressible viscous fluid near a porous infinite plate with arbitrary time dependent heating plate. The effects of chemical reaction in two dimensional steady free convection flow of an electrically conducting viscous fluid through a porous medium bounded by vertical surface with slip flow region has been studied by Senapatil et al. [12]. Khan et al. [13] analyzed the effects of radiation and thermal diffusion on MHD free convection flow of an incompressible viscous fluid near an oscillating plate embedded in a porous medium.

The influence of magnetic field on the other hand is observed in several natural and human-made flows. Magnetic fields are commonly applied in industry to pump, heat, levitate and stir liquid metals. There is the terrestrial magnetic field which is maintained by fluid flow in the earth's core, the solar magnetic field which originates sunspots and solar flares, and the galactic magnetic field which is thought to control the configuration of stars from interstellar clouds [14]. Recently, considerable attention has been focused on applications of MHD and heat transfer such as metallurgical processing, MHD generators and geothermal energy extraction. The phenomenon concerning heat and mass transfer with MHD flow is important due to its numerous applications in science and technology. The particular applications are found in buoyancy induced flows in the atmosphere, in bodies of water and quasi-solid bodies such as earth. Therefore, heat and mass transfer with MHD flow has been a subject of concern of several researchers including Hayat et al. [15], Jha and Apere [16] and Fetecau et al. [17].

Furthermore, it is found from the literature that several investigations on free convection flows are available with different thermal conditions at the bounding plate which are continuous and well-defined at the wall. However, most of the practical problems appear with non-uniform or arbitrary conditions at the wall. To study such problems, it is useful to investigate them under step change in wall temperature. The physical implication of this idea can be found in the fabrication of thin-film photovoltaic devices where ramped wall temperatures may be employed to achieve a specific finish of the system [18]. According to [19], periodic temperature step changes are also important in building heat transfer applications such as in air conditioning, where the conventional assumption of periodic outdoor conditions may lead to considerable errors in the case of a significant temporary deviation of the temperature from periodicity. Keeping this in view, several authors have studied free convection flow past a vertical plate with step discontinuities in the surface temperature. 
However, here we are only highlighting some recent and important contributions [20-25].

On the other hand, the motion of the fluid past an infinite plate is of great interest for academic research due to its various practical applications. Of course such motion can be induced as a results of several effects including motions due to boundaries and applications of the wall shear stress. Exact solutions of the problems with shear stress on the bounding plate are quite complicated and therefore, very few studies are available in the literature. Such studies are even scarce with combined effects of heat and mass transfer. Navier [26] had proposed a slip boundary condition where the slip velocity depends linearly on the shear stress. Generally, the slip velocity strongly depends on the shear stress and mostly governing equations for slip are developed under this assumption. The slip that appears at the wall has led to the study of an interesting class of problems in which the shear stress is given on the solid boundary. Having such motivation in mind, Fetecau et al. [28] investigated free convection flow near a vertical plate that applies arbitrary shear stress to the fluid when the thermal radiation and porosity effects are taken into consideration. However, so far no study has been reported in the literature which focuses on the conjugate free convection flow with ramped wall temperature under the arbitrary shear stress condition. Even such studies are not available for viscous fluids.

Therefore, the aim of the present investigation is to provide exact solutions for MHD conjugate flow of a Newtonian fluid past an infinite plate that applies arbitrary shear stress to the fluid. More exactly, we consider the vertical plate situated in the $(x, z)$ plane of a Cartesian coordinate system $O x y z$, the domain of the flow is the porous half-space $y>0$ and the arbitrary shear stress on the vertical plate is given by $\frac{f(t)}{\mu}$, where $f(t)$ is an arbitrary function and $\mu$ is the viscosity. Closed form solutions of the initial and boundary value problems that govern the flow are obtained by means of the integral transform method. Some special cases are extracted from the general solutions together with some limiting solutions in the literature. The results for velocity, temperature and concentration profiles are plotted graphically and discussed for the embedded flow parameters.

\section{Mathematical Formulation}

Let us consider the unsteady free convection flow of an incompressible viscous fluid over an infinite vertical plate embedded in a porous medium. The physical configuration of the problem is shown in Fig. 1. The $x$-axis is taken along the plate and the $y$-axis is taken normal to it. Initially, both the plate and fluid are at stationary conditions with the constant temperature $T_{\infty}$ and concentration $C_{\infty}$. After time $t=0^{+}$, the plate applies a time dependent shear stress $f(t)$ to the fluid along the $x$-axis. Meanwhile, the temperature of the plate is raised or lowered to $T_{\infty}+\left(T_{w}-T_{\infty}\right) \frac{t}{t_{0}}$ when $t \leq t_{0}$, and thereafter, for $t>t_{0}$, is maintained at constant temperature $T_{w}$ and concentration is raised to $C_{w}$. The radiation terms is also considered in the energy equation. However, the radiative heat flux is considered negligible in $x$-direction compare to $y$-direction. We assume that the flow is laminar and the fluid is grey absorbing-emitting radiation but no scattering medium. In addition to that we asume that the fluid is electrically conducting. Therefore, we use the following Maxwell equations

$$
\operatorname{div} \mathbf{B}=\mathbf{0}, \operatorname{Curl} \mathbf{E}=-\frac{\partial \mathbf{B}}{\partial t}, \operatorname{Curl} \mathbf{B}=\mu_{e} \mathbf{J}
$$

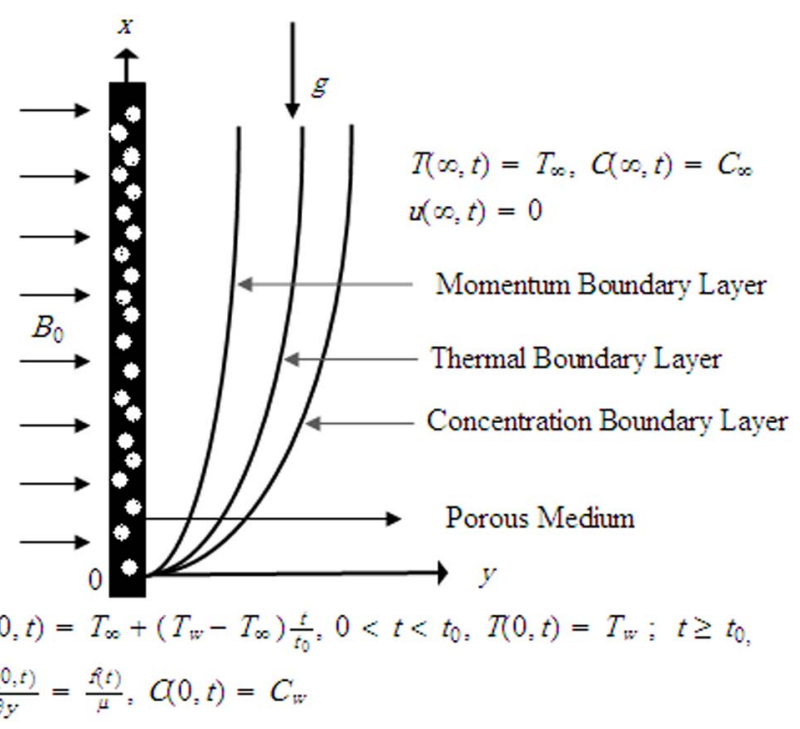

Figure 1. Physical configuration of the problem. doi:10.1371/journal.pone.0090280.g001

In the above equations, $\mathbf{B}, \mathbf{E}$ and $\mu_{e}$ are the magnetic field, electric field intensity and the magnetic permeability of the fluid, respectively. By using Ohm's law, the current density $\mathbf{J}$ is given as

$$
\mathbf{J}=\sigma(\mathbf{E}+\mathbf{V} \times \mathbf{B}),
$$

where $\sigma$ is the electrical conductivity of the fluid. Further we make the following assumptions:

- The quantities $\rho, \mu_{e}$ and $\sigma$ are all constants throughout the flow field.

- The magnetic field $\mathbf{B}$ is perpendicular to the velocity field $\mathbf{V}$.

- The induced magnetic field $\mathbf{b}$ is negligible compared with the imposed magnetic field $\mathbf{B}_{0}$.

- The magnetic Reynolds number is small.

- The electric field is zero.

In view of above assumptions, the electromagnetic body force takes the linearized form [15]

$$
\frac{1}{\rho} \mathbf{J} \times \mathbf{B}=\frac{\sigma}{\rho}\left[\left(\mathbf{V} \times \mathbf{B}_{0}\right) \times \mathbf{B}_{0}\right]=-\frac{\sigma B_{0}^{2} \mathbf{V}}{\rho} .
$$

Using Boussinesq's approximation and neglecting the viscous dissipation, the equations governing the flow are given by $[2,32]$

$$
\frac{\partial u}{\partial t}=v \frac{\partial^{2} u}{\partial y^{2}}+g \beta_{T}\left(T-T_{\infty}\right)+g \beta_{C}\left(C-C_{\infty}\right)-\frac{v}{K} u-\frac{\sigma B_{0}^{2}}{\rho} u ;
$$

$y, t>0$

$$
\rho C_{p} \frac{\partial T}{\partial t}=k \frac{\partial^{2} T}{\partial y^{2}}-\frac{\partial q_{r}}{\partial y} \quad y, t>0
$$

$$
\frac{\partial C}{\partial t}=D \frac{\partial^{2} C}{\partial y^{2}} \quad y, t>0
$$


where $u, T, C, v, \rho, g, \beta_{T}, \beta_{C}, K, \sigma, B_{0}, C_{p}, k, q_{r}$, and $D$ are the velocity of the fluid in $x$-direction, its temperature and concentration, the kinematic viscosity, the constant density, the gravitational acceleration, the heat transfer coefficient, the mass transfer coefficient, the permeability of the porous medium, the electric conductivity of the fluid, the applied magnetic field, the heat capacity at constant pressure, the thermal conductivity, the radiative heat flux and mass diffusivity.

The corresponding initial and boundary conditions are

$$
\begin{aligned}
& u(y, 0)=0, T(y, 0)=T_{\infty}, C(y, 0)=C_{\infty} ; \quad \forall y \geq 0, \\
& \frac{\partial u(0, t)}{\partial y}=\frac{f(t)}{\mu}, C(0, t)=C_{w} ; t>0, \\
& T(0, t)=T_{\infty}+\left(T_{w}-T_{\infty}\right) \frac{t}{t_{0}}, 0<t<t_{0}, T(0, t)=T_{w} ; t \geq t_{0},
\end{aligned}
$$$$
u(\infty, t)=0, T(\infty, t)=T_{\infty}, C(\infty, t)=C_{\infty} ; t>0 .
$$

The radiation heat flux under Rosseland approximation for optically thick fluid $[8,9,29,30,31]$ is given by

$$
q_{r}=-\frac{4 \sigma^{*}}{3 k_{R}} \frac{\partial T^{4}}{\partial y}
$$

where $\sigma^{*}$ and $k_{R}$ are the Stefan-Boltzmann constant and the mean spectral absorption coefficient respectively. It is supposed that the temperature difference within the flow are sufficiently small, then Eq. (8) can be linearized by expanding $T^{4}$ into Taylor series about $T_{\infty}$, and neglecting higher order terms, we find that

$$
T^{4} \approx 4 T_{\infty}^{3} T-3 T_{\infty}^{4}
$$

Substituting Eq. (9) into Eq. (8) and then putting the obtained result in Eq. (5), we get

$$
\operatorname{Pr} \frac{\partial T}{\partial t}=v(1+N r) \frac{\partial^{2} T}{\partial y^{2}} ; \quad y, t>0,
$$

where $\operatorname{Pr}, v$ and $N r$ are defined by

$$
\operatorname{Pr}=\frac{\mu C_{p}}{k}, v=\frac{\mu}{\rho}, N_{r}=\frac{16 \sigma T_{\infty}^{3}}{3 k k_{R}} .
$$

By introducing the following dimensionless variables

$$
\begin{aligned}
& u^{*}=u \sqrt{\frac{t_{0}}{v}}, T^{*}=\frac{T-T_{\infty}}{T_{w}-T_{\infty}}, C^{*}=\frac{C-C_{\infty}}{C_{w}-C_{\infty}}, y^{*}=\frac{y}{\sqrt{v t_{0}}}, \\
& t^{*}=\frac{t}{t_{0}}, f^{*}\left(t^{*}\right)=\frac{t_{\circ}}{\mu} f\left(t_{0} t^{*}\right),
\end{aligned}
$$

into Eqs. (4), (6) and (10) and dropping out the star notations, we get

$$
\begin{gathered}
\frac{\partial u}{\partial t}=\frac{\partial^{2} u}{\partial y^{2}}+G r T+G m C-K_{p} u-M u \\
\operatorname{Pr}_{e f f} \frac{\partial T}{\partial t}=\frac{\partial^{2} T}{\partial y^{2}}, \\
\frac{\partial C}{\partial t}=\frac{1}{S c} \frac{\partial^{2} C}{\partial y^{2}}
\end{gathered}
$$

where $\operatorname{Pr}_{\text {eff }}=\frac{\operatorname{Pr}}{1+N r}$ is the effective Prandtl number [29]; Eq. (10)

$$
\begin{aligned}
& G r=\frac{g \beta_{T}\left(T_{w}-T_{\infty}\right) v}{U_{0}^{3}}, G m=\frac{g \beta_{c}\left(C_{w}-C_{\infty}\right) v}{U_{0}^{3}}, \\
& M=\frac{\sigma B_{0}^{2} t_{0}}{\rho}, S c=\frac{v}{D}, K_{p}=\frac{v t_{0}}{K}, t_{0}=\frac{v}{U_{0}^{2}},
\end{aligned}
$$

are the Grashof number, modified Grashof number, magnetic parameter, Schmidt number, the inverse permeability parameter for the porous medium and the characteristic time respectively.

The corresponding dimensionless initial and boundary conditions are

$$
\begin{aligned}
& u(y, 0)=0, T(y, 0)=0 C(y, 0)=0 ; \forall y \geq 0, \\
& \left.\frac{\partial u}{\partial y}\right|_{y=0}=f(t), T(0, t)=t ; 0<t \leq 1, T(0, t)=1 ; t>1, \\
& C(0, t)=1, C(\infty, t)=0, T(\infty, t)=0, u(\infty, t)=0 ; t>0 .
\end{aligned}
$$

\section{Solution of the Problem}

In order to solve Eqs. (13)-(15) under conditions (16), we use the Laplace transform technique and get the following differential equations

$$
\begin{aligned}
q \bar{u}(y, q)= & \frac{\partial^{2} \bar{u}(y, q)}{\partial y^{2}}+G r \bar{T}(y, q)+G m \bar{C}(y, q) \\
& -K_{p} \bar{u}(y, q)-M \bar{u}(y, q),
\end{aligned}
$$

$$
\begin{gathered}
\bar{T}(y, q)=\frac{1}{\operatorname{Pr}_{e f f} q} \frac{\partial^{2} \bar{T}(y, q)}{\partial y^{2}} \\
\bar{C}(y, q)=\frac{1}{S c q} \frac{\partial^{2} \bar{C}(y, q)}{\partial y^{2}}
\end{gathered}
$$

with boundary conditions 


$$
\begin{aligned}
& \bar{C}(\infty, q)=0, \bar{C}(0, q)=\frac{1}{q}, \bar{T}(\infty, q)=0, \\
& \bar{u}(\infty, q)=0,\left.\frac{\partial \bar{u}(y, q)}{\partial y}\right|_{y=0}=F(q), T(0, q)=\frac{1-e^{-q}}{q^{2}} .
\end{aligned}
$$

Solving Eq. (18) in view of Eq. (20), we get

$$
\bar{T}(y, q)=\frac{1}{q^{2}} e^{-y \sqrt{q \operatorname{Pr}_{e f f}}}-\frac{e^{-q}}{q^{2}} e^{-y \sqrt{q \operatorname{Pr}_{e f f}}},
$$

which upon inverse Laplace transform gives

where

$$
T(y, t)=f(y, t)-f(y, t-1) H(t-1),
$$

$$
\begin{aligned}
f(y, t)= & \left(\frac{\operatorname{Pr}_{e f f} y^{2}}{2}+t\right) \operatorname{erf} c\left(\frac{\sqrt{\operatorname{Pr}_{e f f} y}}{2 \sqrt{t}}\right) \\
& -\sqrt{\frac{\operatorname{Pr}_{\text {eff }} t}{\pi}} y \exp \left(\frac{-\operatorname{Pr}_{e f f} y^{2}}{4 t}\right)
\end{aligned}
$$

and

$$
\left.\frac{\partial T(y, t)}{\partial y}\right|_{y=0}=\frac{2 \sqrt{\operatorname{Pr}_{e f f}}}{\sqrt{\pi}}(\sqrt{t}-\sqrt{t-1} H(t-1)),
$$

is the corresponding heat transfer rate also known as Nusselt number. Here $\operatorname{erf}($.$) and erf c($.$) denote the error function and$ complementary error function of Gauss [28].

Solution of Eq. (19) using boundary conditions from Eq. (20) yields

$$
C(y, q)=\frac{1}{q} e^{-y \sqrt{S c q}}
$$

which upon inverse Laplace transform gives

and

$$
C(y, t)=\operatorname{erf} c\left(\frac{y \sqrt{S c}}{2 \sqrt{t}}\right)
$$

$$
\left.\frac{\partial C(y, t)}{\partial y}\right|_{y=0}=-\frac{\sqrt{S c}}{\sqrt{\pi t}}
$$

is the corresponding mass transfer rate also known as Sherwood number.

The solution of Eq. (17) under boundary conditions (20) results

$$
\begin{aligned}
\bar{u}(y, q)= & \frac{a_{1} \sqrt{q}}{q^{2}\left(q-a_{2}\right) \sqrt{q+H_{1}}} e^{-y \sqrt{q+H_{1}}} \\
& -\frac{a_{1} \sqrt{q} e^{-q}}{q^{2}\left(q-a_{2}\right) \sqrt{q+H_{1}}} e^{-y \sqrt{q+H_{1}}} \\
& -\frac{F(q)}{\sqrt{q+H_{1}}} e^{-y \sqrt{q+H_{1}}}-\frac{a_{3}}{q^{2}\left(q-a_{2}\right)} e^{-y \sqrt{q \operatorname{Pr}_{e f f}}} \\
& +\frac{a_{3} e^{-q}}{q^{2}\left(q-a_{2}\right)} e^{-y \sqrt{q \operatorname{Pr}_{e f f}}} \\
& +\frac{a_{4} \sqrt{q}}{q\left(q-a_{5}\right) \sqrt{q+H_{1}}} e^{-y \sqrt{q+H_{1}}}-\frac{a_{6}}{q\left(q-a_{5}\right)} e^{-y \sqrt{q S c}},
\end{aligned}
$$

which upon inverse Laplace transform results

$$
u(y, t)=u_{c}(y, t)+u_{m}(y, t),
$$

where

$$
\begin{aligned}
& u_{c}(y, t)=a_{1} \int_{0}^{t}\left(\frac{e^{a_{2}(t-s)} \operatorname{erf}\left(\sqrt{a_{2}(t-s)}\right)}{\left(a_{2}\right)^{\frac{3}{2}}}-\frac{2 \sqrt{t-s}}{\sqrt{\pi} a_{2}}\right) \frac{e^{-H_{1} s-\frac{y^{2}}{4 s}}}{\sqrt{\pi s}} d s \\
& +\left[\frac{a_{1}}{a_{2} \pi} \int_{0}^{t-1} \frac{(2 \sqrt{t-1-s}) e^{-H_{1} s-\frac{y^{2}}{4 s}}}{\sqrt{s}} d s\right] H(t-1) \\
& -\left[\frac{a_{1}}{\left(a_{2}\right)^{\frac{3}{2}} \sqrt{\pi}} \int_{0}^{t-1} \frac{e^{a_{2}(t-1-s)-H_{1} s-\frac{y^{2}}{4 s}} \operatorname{erf}\left(\sqrt{a_{2}(t-1-s)}\right)}{\sqrt{s}} d s\right] H(t-1) \\
& +a_{4} \int_{0}^{t}\left(\frac{e^{a_{5}(t-s)} \operatorname{erf}\left(\sqrt{a_{5}(t-s)}\right)}{\sqrt{a_{5}}}-\frac{2 \sqrt{t-s}}{\sqrt{\pi} a_{2}}\right) \frac{e^{-H_{1} s-\frac{y^{2}}{4 s}}}{\sqrt{\pi s}} d s \\
& +\frac{a_{3}}{a_{2}}\left(t+\frac{\operatorname{Pr}_{e f f} y^{2}}{2}\right) \operatorname{erf} c\left(\frac{y \sqrt{\operatorname{Pr}_{e f f}}}{2 \sqrt{t}}\right)
\end{aligned}
$$

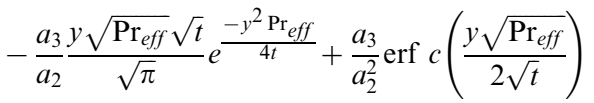

$$
\begin{aligned}
& -\frac{a_{3} e^{a_{2} t+y} \sqrt{\mathrm{Pr}_{e f f} a_{2}}}{2 a_{2}^{2}} \operatorname{erf} c\left(\frac{y \sqrt{\operatorname{Pr}_{e f f}}}{2 \sqrt{t}}+\sqrt{a_{2} t}\right) \\
& -\frac{a_{3} e^{a_{2} t-y \sqrt{\operatorname{Pr}_{e f f} a_{2}}}}{2 a_{2}^{2}} \operatorname{erf} c\left(\frac{y \sqrt{\operatorname{Pr}_{e f f}}}{2 \sqrt{t}}-\sqrt{a_{2} t}\right) \\
& -\frac{a_{3}}{a_{2}}\left((t-1)+\frac{\operatorname{Pr}_{e f f} y^{2}}{2}\right) \operatorname{erf} c\left(\frac{y \sqrt{\operatorname{Pr}_{e f f}}}{2 \sqrt{t-1}}\right) H(t-1) \\
& +\frac{a_{3}}{a_{2}} \frac{y \sqrt{\operatorname{Pr}_{e f f}} \sqrt{t-1}}{\sqrt{\pi}} e^{\frac{-y^{2} \operatorname{Pr}_{e f f}}{4(t-1)}} H(t-1) \\
& -\frac{a_{3}}{a_{2}^{2}} \operatorname{erf} c\left(\frac{y \sqrt{\operatorname{Pr}_{e f f}}}{2 \sqrt{t-1}}\right) H(t-1)+\frac{a_{6}}{a_{5}} \operatorname{erf} c\left(\frac{y \sqrt{S c}}{2 \sqrt{t}}\right)
\end{aligned}
$$

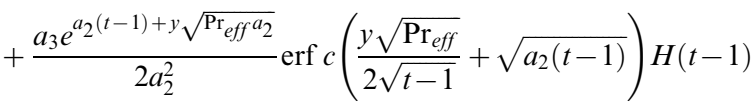

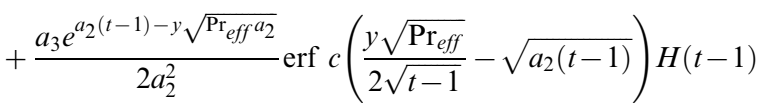

$$
\begin{aligned}
& -\frac{a_{6} e^{a_{5} t-y \sqrt{a_{5} S c}}}{2 a_{5}} \operatorname{erf} c\left(\frac{y \sqrt{S c}}{2 \sqrt{t}}-\sqrt{a_{5} t}\right) \\
& -\frac{a_{6} e^{a_{5} t+y \sqrt{a_{5} S c}}}{2 a_{5}} \operatorname{erf} c\left(\frac{y \sqrt{S c}}{2 \sqrt{t}}+\sqrt{a_{5} t}\right)
\end{aligned}
$$

and

$$
u_{m}(y, t)=-\frac{1}{\sqrt{\pi}} \int_{0}^{t} \frac{f(t-s) e^{-H_{1} s-\frac{y^{2}}{4 s}}}{\sqrt{s}},
$$

correspond to the convective and mechanical parts of velocity. 
It is noted from Eqs. (22) and (30) that $T(y, t)$ is valid for all positive values of $\operatorname{Pr}_{\text {eff }}$ while the $u_{c}(y, t)$ is not valid for $\operatorname{Pr}_{\text {eff }}=1$. Therefore, to get $u_{c}(y, t)$ when the effective Prandtl number is not equal to one, we make $\operatorname{Pr}_{\text {eff }}=1$ into Eq. (14), use a similar procedure as discussed above, and obtain

$$
\begin{aligned}
\bar{u}(y, q)= & \frac{-a_{14}}{q^{\frac{3}{2}} \sqrt{q+H_{1}}} e^{-y \sqrt{q+H_{1}}}+\frac{a_{14} e^{-q}}{q^{\frac{3}{2}} \sqrt{q+H_{1}}} e^{-y \sqrt{q+H_{1}}} \\
& -\frac{F(q)}{\sqrt{q+H_{1}}} e^{-y \sqrt{q+H_{1}}}+\frac{a_{14}}{q^{2}} e^{-y \sqrt{q}}-\frac{a_{14} e^{-q}}{q^{2}} e^{-y \sqrt{q}} \\
& +\frac{a_{4} \sqrt{q}}{q\left(q-a_{5}\right) \sqrt{q+H_{1}}} e^{-y \sqrt{q+H_{1}}}-\frac{a_{6}}{q\left(q-a_{5}\right)} e^{-y \sqrt{q S c}} .
\end{aligned}
$$

By taking inverse Laplace transform we find that

$$
\begin{aligned}
& u(y, t)=-\frac{2 a_{14}}{\pi} \int_{0}^{t} \frac{\sqrt{t-s} e^{-H_{1} s-\frac{y^{2}}{4 s}}}{\sqrt{s}} d s \\
& +\left(\frac{2 a_{14}}{\pi} \int_{0}^{t-1} \frac{\sqrt{t-1-s} e^{-H_{1} s-\frac{y^{2}}{4 s}}}{\sqrt{s}} d s\right) H(t-1) \\
& +a_{4} \int_{0}^{t}\left(\frac{e^{a_{5}(t-s)} \operatorname{erf}\left(\sqrt{a_{5}(t-s)}\right)}{\sqrt{a_{5}}}-\frac{2 \sqrt{t-s}}{\sqrt{\pi} a_{2}}\right) \frac{e^{-H_{1} s-\frac{y^{2}}{4 s}}}{\sqrt{\pi s}} d s \\
& +a_{14}\left[\left(t+\frac{y^{2}}{2}\right) \operatorname{erf} c\left(\frac{y}{2 \sqrt{t}}\right)-\frac{y \sqrt{t}}{\sqrt{\pi}} e^{\frac{-y^{2}}{4 t}}\right] \\
& -\frac{a_{6} e^{a_{5} t-y \sqrt{a_{5} S c}}}{2 a_{5}} \operatorname{erf} c\left(\frac{y \sqrt{S c}}{2 \sqrt{t}}-\sqrt{a_{5} t}\right) \\
& -a_{14}\left[\left(t-1+\frac{y^{2}}{2}\right) \operatorname{erf} c\left(\frac{y}{2 \sqrt{t-1}}\right)-\frac{y \sqrt{t-1}}{\sqrt{\pi}} e^{\frac{-y^{2}}{4(t-1)}}\right] H(t-1) \\
& +\frac{a_{6}}{a_{5}} \operatorname{erf} c\left(\frac{y \sqrt{S c}}{2 \sqrt{t}}\right)-\frac{1}{\sqrt{\pi}} \int_{0}^{t} \frac{f(t-s) e^{-H_{1} s-\frac{y^{2}}{4 s}}}{\sqrt{s}} d s \\
& -\frac{a_{6} e^{a_{5} t+y \sqrt{a_{5} S c}} \operatorname{erf} c\left(\frac{y \sqrt{S c}}{2 \sqrt{t}}+\sqrt{a_{5} t}\right)}{2 a_{5}}
\end{aligned}
$$

where

$$
\begin{aligned}
& a_{1}=\frac{G r \sqrt{\operatorname{Pr}_{e f f}}}{\operatorname{Pr}_{e f f}-1}, a_{2}=\frac{H_{1}}{\operatorname{Pr}_{e f f}-1}, a_{3}=\frac{G r}{\operatorname{Pr}_{e f f}-1}, a_{4}=\frac{G m \sqrt{S c}}{S c-1}, \\
& a_{5}=\frac{H_{1}}{S c-1}, a_{6}=\frac{G m}{S c-1}, a_{7}=\frac{G r \sqrt{\operatorname{Pr}}}{\operatorname{Pr}-1}, a_{8}=\frac{H_{1}}{\operatorname{Pr}-1}, \\
& a_{9}=\frac{G r}{\operatorname{Pr}-1}, a_{10}=\frac{K_{p}}{\operatorname{Pr}_{e f f}-1}, a_{11}=\frac{K_{p}}{S c-1}, a_{12}=\frac{M}{\operatorname{Pr}_{e f f}-1}, \\
& a_{13}=\frac{M}{S c-1}, a_{14}=\frac{G r}{H_{1}}, H_{1}=K_{p}+M .
\end{aligned}
$$

\section{Plate with Constant Temperature}

Equations (22) and (29) give analytical expressions for the temperature and velocity near a vertical plate with ramped temperature. In order to highlight the effect of the ramped temperature distribution of the boundary on the flow, it is important to compare such a flow with the one near a plate with constant temperature. It can be shown that the temperature, rate of heat transfer and velocity for the flow near an isothermal plate are

$$
\begin{gathered}
T(y, t)=\operatorname{erf} c\left(\frac{y \sqrt{\operatorname{Pr}_{e f f}}}{2 \sqrt{t}}\right), \\
\frac{\partial T(0, t)}{\partial y}=-\frac{\sqrt{\operatorname{Pr}_{e f f}}}{\sqrt{\pi t}},
\end{gathered}
$$

$$
\begin{aligned}
& u_{c}(y, t)=\frac{a_{1}}{\sqrt{\pi a_{2}}} \int_{0}^{t} \frac{e^{a_{2}(t-s)-H_{1} s-\frac{y^{2}}{4 s}} \operatorname{erf}\left(\sqrt{a_{2}(t-s)}\right)}{\sqrt{s}} d s \\
& +\frac{a_{4}}{\sqrt{\pi a_{5}}} \int_{0}^{t} \frac{e^{a_{5}(t-s)-H_{1} s-\frac{y^{2}}{4 s}} \operatorname{erf}\left(\sqrt{a_{5}(t-s)}\right)}{\sqrt{s}} d s
\end{aligned}
$$

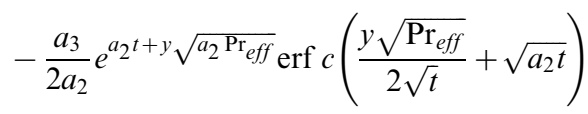

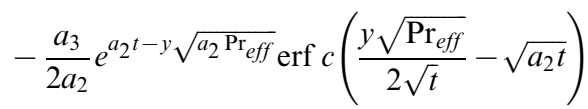

$$
\begin{aligned}
& -\frac{a_{6}}{2 a_{5}} e^{a_{5} t-y \sqrt{a_{5} S c}} \operatorname{erf} c\left(\frac{y \sqrt{S c}}{2 \sqrt{t}}-\sqrt{a_{5} t}\right) \\
& +\frac{a_{3}}{a_{2}} \operatorname{erf} c\left(\frac{y \sqrt{\operatorname{Pr}_{e f f}}}{2 \sqrt{t}}\right)+\frac{a_{6}}{a_{5}} \operatorname{erf} c\left(\frac{y \sqrt{S c}}{2 \sqrt{t}}\right) \\
& -\frac{a_{6}}{2 a_{5}} e^{a_{5} t+y \sqrt{a_{5} S c}} \operatorname{erf} c\left(\frac{y \sqrt{S c}}{2 \sqrt{t}}+\sqrt{a_{5} t}\right), \\
& u_{m}(y, t)=-\frac{1}{\sqrt{\pi}} \int_{0}^{t} \frac{f(t-s) e^{-H_{1} s-\frac{y^{2}}{4 s}}}{\sqrt{s}} d s .
\end{aligned}
$$

As previously, Eq. (37) is not valid for $\operatorname{Pr}_{\text {eff }}=1$. Therefore we calculate separately solution for velocity by taking $\operatorname{Pr}_{\text {eff }}=1$ into Eq. (14) and finally get

$$
\begin{aligned}
u(y, t)= & a_{14} \operatorname{erf} c\left(\frac{y}{2 \sqrt{t}}\right)-\frac{a_{14}}{\sqrt{\pi}} \int_{0}^{t} \frac{e^{-H_{1} s-\frac{y^{2}}{4 s}}}{\sqrt{(t-s) s}} d s \\
& +\frac{a_{4}}{\sqrt{\pi a_{5}}} \int_{0}^{t} \frac{e^{a_{5}(t-s)-H_{1} s-\frac{y^{2}}{4 s} \operatorname{erf}\left(\sqrt{a_{5}(t-s)}\right)}}{\sqrt{s}} d s \\
& +\frac{a_{6}}{a_{5}} \operatorname{erf} c\left(\frac{y \sqrt{S c}}{2 \sqrt{t}}\right)-\frac{1}{\sqrt{\pi}} \int_{0}^{t} \frac{f(t-s) e^{-H_{1} s-\frac{y^{2}}{4 s}}}{\sqrt{s}} d s \\
& -\frac{a_{6}}{2 a_{5}} e^{a_{5} t+y \sqrt{a_{5} S c}} \operatorname{erf} c\left(\frac{y \sqrt{S c}}{2 \sqrt{t}}+\sqrt{a_{5} t}\right) \\
& -\frac{a_{6}}{2 a_{5}} e^{a_{5} t-y \sqrt{a_{5} S c}} \operatorname{erf} c\left(\frac{y \sqrt{S c}}{2 \sqrt{t}}-\sqrt{a_{5} t}\right)
\end{aligned}
$$




\section{Limiting Cases}

In this section we discuss few limiting cases of our general solutions.

5.1 Solution in the absence of porous effects for ramped and constant wall temperature $\left(K_{p} \rightarrow 0\right)$

$u(y, t)=a_{1} \int_{0}^{t}\left(\frac{e^{a_{12}(t-s)} \operatorname{erf}\left(\sqrt{a_{12}(t-s)}\right)}{\left(a_{12}\right)^{\frac{3}{2}}}-\frac{2 \sqrt{t-s}}{\sqrt{\pi} a_{12}}\right) \frac{e^{-M s-\frac{y^{2}}{4 s}}}{\sqrt{\pi s}} d s$

$+\left[\frac{a_{1}}{a_{12} \pi} \int_{0}^{t-1} \frac{(2 \sqrt{t-1-s}) e^{-M s-\frac{y^{2}}{4 s}}}{\sqrt{s}} d s\right] H(t-1)$

$-\left[\frac{a_{1}}{\left(a_{12}\right)^{\frac{3}{2}} \sqrt{\pi}} \int_{0}^{t-1} \frac{e^{a_{12}(t-1-s)-M s-\frac{y^{2}}{4 s}} \operatorname{erf}\left(\sqrt{a_{12}(t-1-s)}\right)}{\sqrt{s}} d s\right] H(t-1)$

$+a_{4} \int_{0}^{t}\left(\frac{e^{a_{13}(t-s)} \operatorname{erf}\left(\sqrt{a_{13}(t-s)}\right)}{\sqrt{a_{13}}}-\frac{2 \sqrt{t-s}}{\sqrt{\pi} a_{12}}\right) \frac{e^{-M s-\frac{y^{2}}{4 s}}}{\sqrt{\pi s}} d s$

$+\frac{a_{3}}{a_{12}}\left(t+\frac{\operatorname{Pr}_{e f f} y^{2}}{2}\right) \operatorname{erf} c\left(\frac{y \sqrt{\operatorname{Pr}_{e f f}}}{2 \sqrt{t}}\right)-\frac{1}{\sqrt{\pi}} \int_{0}^{t} \frac{f(t-s) e^{-M s-\frac{y^{2}}{4 s}}}{\sqrt{s}} d s$

$-\frac{a_{3}}{a_{12}} \frac{y \sqrt{\operatorname{Pr}_{e f f}} \sqrt{t}}{\sqrt{\pi}} e^{\frac{-y^{2} \operatorname{Pr}_{e f f}}{4 t}}+\frac{a_{3}}{a_{12}^{2}} \operatorname{erf} c\left(\frac{y \sqrt{\operatorname{Pr}_{e f f}}}{2 \sqrt{t}}\right)$

$-\frac{a_{3} e^{a_{12} t+y \sqrt{\operatorname{Pr}_{e f f} a_{12}}}}{2 a_{12}^{2}} \operatorname{erf} c\left(\frac{y \sqrt{\operatorname{Pr}_{e f f}}}{2 \sqrt{t}}+\sqrt{a_{12} t}\right)$

$-\frac{a_{3} e^{a_{12} t-y \sqrt{\operatorname{Pr}_{e f f} a_{12}}}}{2 a_{12}^{2}} \operatorname{erf} c\left(\frac{y \sqrt{\operatorname{Pr}_{e f f}}}{2 \sqrt{t}}-\sqrt{a_{12} t}\right)$

$-\frac{a_{3}}{a_{12}}\left((t-1)+\frac{\operatorname{Pr}_{e f f} y^{2}}{2}\right) \operatorname{erf} c\left(\frac{y \sqrt{\operatorname{Pr}_{e f f}}}{2 \sqrt{t-1}}\right) H(t-1)$

$+\frac{a_{3}}{a_{12}} \frac{y \sqrt{\operatorname{Pr}_{e f f}} \sqrt{t-1}}{\sqrt{\pi}} e^{\frac{-y^{2} \operatorname{Pr}_{e f f}}{4(t-1)}} H(t-1)$

$-\frac{a_{6} e^{a_{13} t-y \sqrt{a_{13} S c}}}{2 a_{13}} \operatorname{erf} c\left(\frac{y \sqrt{S c}}{2 \sqrt{t}}-\sqrt{a_{13} t}\right)$

$-\frac{a_{3}}{a_{12}^{2}} \operatorname{erf} c\left(\frac{y \sqrt{\operatorname{Pr}_{e f f}}}{2 \sqrt{t-1}}\right) H(t-1)+\frac{a_{6}}{a_{13}} \operatorname{erf} c\left(\frac{y \sqrt{S c}}{2 \sqrt{t}}\right)$

$+\frac{a_{3} e^{a_{12}(t-1)+y \sqrt{\operatorname{Pr}_{e f f} a_{12}}}}{2 a_{12}^{2}} \operatorname{erf} c\left(\frac{y \sqrt{\operatorname{Pr}_{e f f}}}{2 \sqrt{t-1}}+\sqrt{a_{12}(t-1)}\right) H(t-1)$

$+\frac{a_{3} e^{a_{12}(t-1)-y \sqrt{\operatorname{Pr}_{e f f} a_{12}}}}{2 a_{12}^{2}} \operatorname{erf} c\left(\frac{y \sqrt{\operatorname{Pr}_{e f f}}}{2 \sqrt{t-1}}-\sqrt{a_{12}(t-1)}\right) H(t-1)$

$-\frac{a_{6} e^{a_{13} t+y \sqrt{a_{13} S c}}}{2 a_{13}} \operatorname{erf} c\left(\frac{y \sqrt{S c}}{2 \sqrt{t}}+\sqrt{a_{13} t}\right)$

$$
\begin{aligned}
& u(y, t)=\frac{a_{1}}{\sqrt{\pi a_{12}}} \int_{0}^{t} \frac{e^{a_{12}(t-s)-M s-\frac{y^{2}}{4 s}} \operatorname{erf}\left(\sqrt{a_{12}(t-s)}\right)}{\sqrt{s}} d s \\
& +\frac{a_{4}}{\sqrt{\pi a_{13}}} \int_{0}^{t} \frac{e^{a_{13}(t-s)-M s-\frac{y^{2}}{4 s}} \operatorname{erf}\left(\sqrt{a_{13}(t-s)}\right)}{\sqrt{s}} d s \\
& -\frac{a_{6}}{2 a_{13}} e^{a_{13} t-y \sqrt{a_{13} S c}} \operatorname{erf} c\left(\frac{y \sqrt{S c}}{2 \sqrt{t}}-\sqrt{a_{13} t}\right)
\end{aligned}
$$

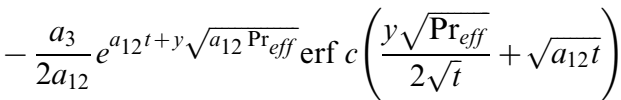

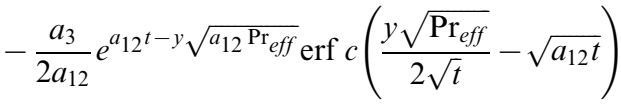

$$
\begin{aligned}
& +\frac{a_{3}}{a_{12}} \operatorname{erf} c\left(\frac{y \sqrt{\operatorname{Pr}_{e f f}}}{2 \sqrt{t}}\right)+\frac{a_{6}}{a_{13}} \operatorname{erf} c\left(\frac{y \sqrt{S c}}{2 \sqrt{t}}\right) \\
& -\frac{a_{6}}{2 a_{13}} e^{a_{13} t+y \sqrt{a_{13} S c}} \operatorname{erf} c\left(\frac{y \sqrt{S c}}{2 \sqrt{t}}+\sqrt{a_{13} t}\right) \text {. }
\end{aligned}
$$

5.2 Solution in the absence of thermal radiation $(\mathrm{Nr} \rightarrow 0)$

In the absence of thermal radiation, the corresponding solutions for ramped and constant wall temperature are directly obtained from the general solutions (22), (24), (29) and (35)-(37) by taking $N r \rightarrow 0$ and replacing $\operatorname{Pr}_{\text {eff }}$ by $\operatorname{Pr}$ i.e.

$u(y, t)=a_{7} \int_{0}^{t}\left(\frac{e^{a_{8}(t-s)} \operatorname{erf}\left(\sqrt{a_{8}(t-s)}\right)}{\left(a_{8}\right)^{\frac{3}{2}}}-\frac{2 \sqrt{t-s}}{\sqrt{\pi} a_{8}}\right) \frac{e^{-H_{1} s-\frac{y^{2}}{4 s}}}{\sqrt{\pi s}} d s$

$+\left[\frac{a_{7}}{\pi a_{8}} \int_{0}^{t-1} \frac{2 \sqrt{t-1-s} e^{-H_{1} s-\frac{y^{2}}{4 s}}}{\sqrt{s}} d s\right] H(t-1)$

$-\left[\frac{a_{7}}{\left(a_{8}\right)^{\frac{3}{2}} \sqrt{\pi}} \int_{0}^{t-1} \frac{\operatorname{erf}\left(\sqrt{a_{8}(t-1-s)}\right) e^{a_{8}(t-1-s)-H_{1} s-\frac{y^{2}}{4 s}}}{\sqrt{s}} d s\right] H(t-1)$

$+a_{4} \int_{0}^{t}\left(\frac{e^{a_{5}(t-s)} \operatorname{erf}\left(\sqrt{a_{5}(t-s)}\right)}{\sqrt{a_{5}}}-\frac{2 \sqrt{t-s}}{\sqrt{\pi} a_{8}}\right) \frac{e^{-H_{1} s-\frac{y^{2}}{4 s}}}{\sqrt{\pi s}} d s$

$+\frac{a_{9}}{a_{8}}\left(t+\frac{\operatorname{Pr} y^{2}}{2}\right) \operatorname{erf} c\left(\frac{y \sqrt{\operatorname{Pr}}}{2 \sqrt{t}}\right)-\frac{1}{\sqrt{\pi}} \int_{0}^{t} \frac{f(t-s) e^{-H_{1} s-\frac{y^{2}}{4 s}}}{\sqrt{s}} d s$

$-\frac{a_{9}}{a_{8}} \frac{y \sqrt{\operatorname{Pr}} \sqrt{t}}{\sqrt{\pi}} e^{\frac{-y^{2} \operatorname{Pr}}{4 t}}+\frac{a_{9}}{a_{8}^{2}} \operatorname{erf} c\left(\frac{y \sqrt{\operatorname{Pr}}}{2 \sqrt{t}}\right)$

$-\frac{a_{9} e^{a_{8} t+y \sqrt{\operatorname{Pr} a_{8}}}}{2 a_{8}^{2}} \operatorname{erf} c\left(\frac{y \sqrt{\operatorname{Pr}}}{2 \sqrt{t}}+\sqrt{a_{8} t}\right)$

$-\frac{a_{9} e^{a_{8} t-y \sqrt{\operatorname{Pr} a_{8}}}}{2 a_{8}^{2}} \operatorname{erf} c\left(\frac{y \sqrt{\operatorname{Pr}}}{2 \sqrt{t}}-\sqrt{a_{8} t}\right)$ 


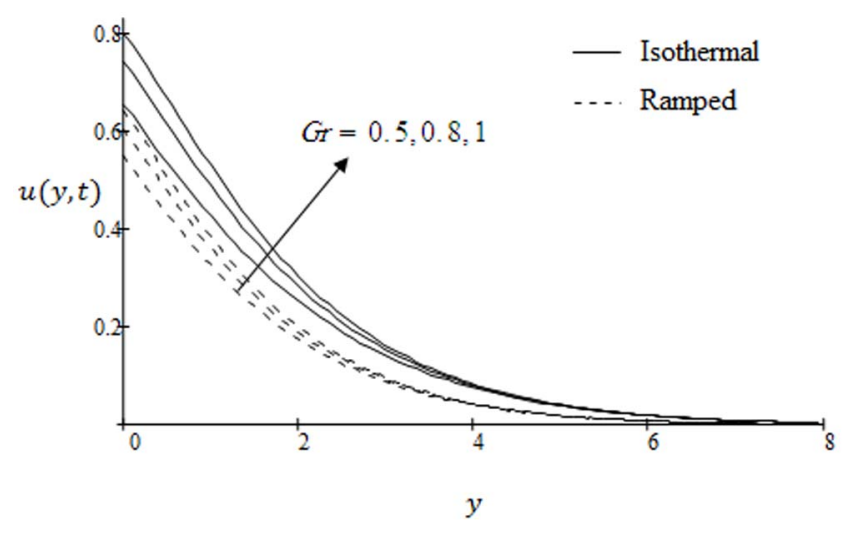

Figure 2. Velocity profiles for different values of $G r$ when the plate applies a constant shear stress $f=-0.25$.

doi:10.1371/journal.pone.0090280.g002

$$
\begin{aligned}
& -\frac{a_{9}}{a_{8}}\left((t-1)+\frac{\operatorname{Pr} y^{2}}{2}\right) \operatorname{erf} c\left(\frac{y \sqrt{\operatorname{Pr}}}{2 \sqrt{t-1}}\right) H(t-1) \\
& -\frac{a_{9}}{a_{8}} \operatorname{erf} c\left(\frac{y \sqrt{\operatorname{Pr}}}{2 \sqrt{t-1}}\right) H(t-1)+\frac{a_{9}}{a_{8}} \frac{y \sqrt{\operatorname{Pr}} \sqrt{t-1}}{\sqrt{\pi}} e^{\frac{-y^{2} \operatorname{Pr}}{4(t-1)}} H(t-1) \\
& +\frac{a_{9} e^{a_{8}(t-1)+y \sqrt{\operatorname{Pr} a_{8}}}}{2 a_{8}^{2}} \operatorname{erf} c\left(\frac{y \sqrt{\operatorname{Pr}}}{2 \sqrt{t-1}}+\sqrt{a_{8}(t-1)}\right) H(t-1) \\
& +\frac{a_{9} e^{a_{12}(t-1)-y \sqrt{\operatorname{Pr} a_{12}}}}{2 a_{8}^{2}} \operatorname{erf} c\left(\frac{y \sqrt{\operatorname{Pr}}}{2 \sqrt{t-1}}-\sqrt{a_{8}(t-1)}\right) H(t-1) \\
& -\frac{a_{6} e^{a_{5} t+y \sqrt{a_{5} S c}}}{2 a_{5}} \operatorname{erf} c\left(\frac{y \sqrt{S c}}{2 \sqrt{t}}+\sqrt{a_{5} t}\right)+\frac{a_{6}}{a_{5}} \operatorname{erf} c\left(\frac{y \sqrt{S c}}{2 \sqrt{t}}\right) \\
& -\frac{a_{6} e^{a_{5} t-y \sqrt{a_{5} S c}}}{2 a_{5}} \operatorname{erf} c\left(\frac{y \sqrt{S c}}{2 \sqrt{t}}-\sqrt{a_{5} t}\right)
\end{aligned}
$$$$
T(y, t)=f_{1}(y, t)-f_{1}(y, t-1) H(t-1)
$$

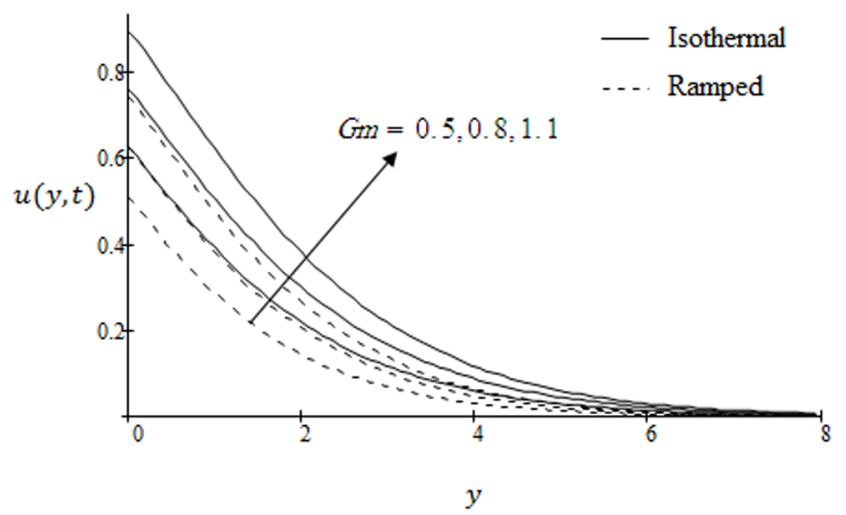

Figure 3. Velocity profiles for different values of $G m$ when the plate applies a constant shear stress $f=-0.25$. doi:10.1371/journal.pone.0090280.g003

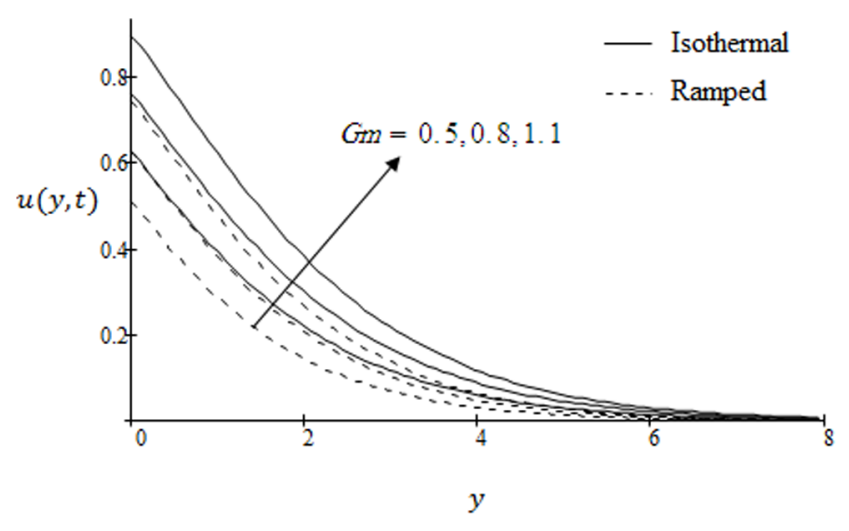

Figure 4. Velocity profiles for different values of $S c$ when the plate applies a constant shear stress $\boldsymbol{f}=-\mathbf{0 . 2 5}$. doi:10.1371/journal.pone.0090280.g004

where

$f_{1}(y, t)=\left(\frac{\operatorname{Pr} y^{2}}{2}+t\right) \operatorname{erf} c\left(\frac{\sqrt{\operatorname{Pr}} y}{2 \sqrt{t}}\right)-\sqrt{\frac{\operatorname{Pr} t}{\pi}} y \exp \left(\frac{-\operatorname{Pr} y^{2}}{4 t}\right)$ and

$$
\left.\frac{\partial T(y, t)}{\partial y}\right|_{y=0}=\frac{2 \sqrt{\operatorname{Pr}}}{\sqrt{\pi}}(\sqrt{t}-\sqrt{t-1} H(t-1)) .
$$

$$
\begin{aligned}
& u(y, t)=\frac{a_{7}}{\sqrt{\pi a_{8}}} \int_{0}^{t} \frac{e^{a_{8}(t-s)-H_{1} s-\frac{y^{2}}{4 s}} \operatorname{erf}\left(\sqrt{a_{8}(t-s)}\right)}{\sqrt{s}} d s \\
& +\frac{a_{4}}{\sqrt{\pi a_{5}}} \int_{0}^{t} \frac{e^{a_{5}(t-s)-H_{1} s-\frac{y^{2}}{4 s} \operatorname{erf}\left(\sqrt{a_{5}(t-s)}\right)}}{\sqrt{s}} d s \\
& -\frac{a_{9}}{2 a_{8}} e^{a_{8} t+y \sqrt{a_{8} \operatorname{Pr}}} \operatorname{erf} c\left(\frac{y \sqrt{\operatorname{Pr}}}{2 \sqrt{t}}+\sqrt{a_{8} t}\right) \\
& -\frac{a_{9}}{2 a_{8}} e^{a_{8} t-y \sqrt{a_{8} \operatorname{Pr}}} \operatorname{erf} c\left(\frac{y \sqrt{\operatorname{Pr}}}{2 \sqrt{t}}-\sqrt{a_{8} t}\right) \\
& +\frac{a_{9}}{a_{8}} \operatorname{erfc}\left(\frac{y \sqrt{\operatorname{Pr}}}{2 \sqrt{t}}\right)+\frac{a_{6}}{a_{5}} \operatorname{erf} c\left(\frac{y \sqrt{S c}}{2 \sqrt{t}}\right) \\
& -\frac{a_{6}}{2 a_{5}} e^{a_{5} t+y \sqrt{a_{5} S c}} \operatorname{erf} c\left(\frac{y \sqrt{S c}}{2 \sqrt{t}}+\sqrt{a_{5} t}\right) \\
& -\frac{a_{6}}{2 a_{5}} e^{a_{5} t-y \sqrt{a_{5} S c} \operatorname{erf} c\left(\frac{y \sqrt{S c}}{2 \sqrt{t}}-\sqrt{a_{5} t}\right)} \\
& -\frac{1}{\sqrt{\pi}} \int_{0}^{t} \frac{f(t-s) e^{-H_{1} s-\frac{y^{2}}{4 s}} d s}{\sqrt{s}}
\end{aligned}
$$

$$
T(y, t)=\operatorname{erf} c\left(\frac{y \sqrt{\operatorname{Pr}}}{2 \sqrt{t}}\right)
$$




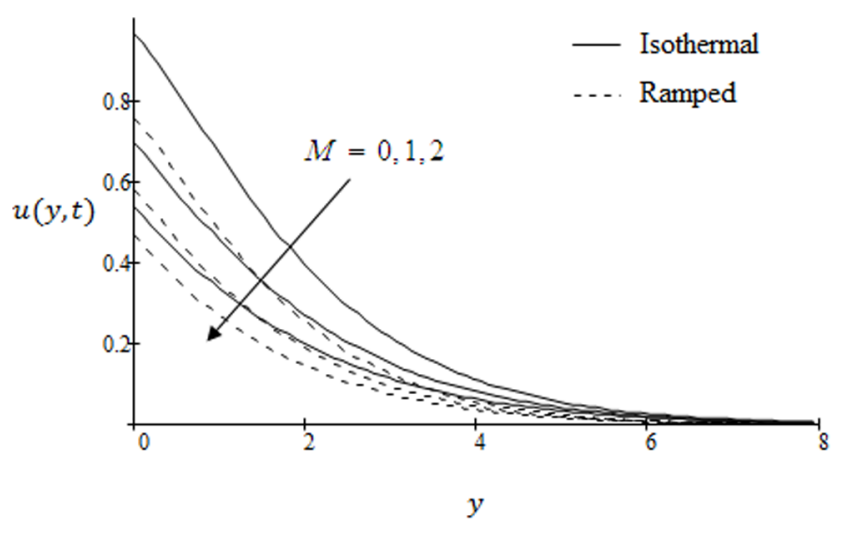

Figure 5. Velocity profiles for different values of $M$ when the plate applies a constant shear stress $f=-0.25$.

doi:10.1371/journal.pone.0090280.g005

$$
\frac{\partial T(0, t)}{\partial y}=-\frac{\sqrt{\operatorname{Pr}}}{\sqrt{\pi t}}
$$

\subsection{Solutions in the absence of free convection}

Let us assume that the flow is caused only due to bounding plate and the corresponding buoyancy forces are zero equivalently it shows the absence of free convection due to the differences in temperature and mass gradients i.e. the terms $G r$ and $G m$ are zero. This shows that the convective parts of velocities are zero in both cases of ramped wall and constant temperature and the flow is only governed by the mechanical part of velocities given by Eqs. (31) and (37).

\subsection{Solutions in the absence of mechanical effects}

In this case we assume that the infinite plate is in static position at every time i.e. the function $f(t)$ is zero for all values of $t$ and the mechanical parts for both ramped and constant wall temperature are equivalently zero. In such a situation, the motion in the fluid is induced only due to the free convection which causes due to the buoyancy forces. Therefore, the velocities of the fluid in both cases of ramped and constant wall temperature are only represented by their convective parts given by Eqs. (30) and (37).

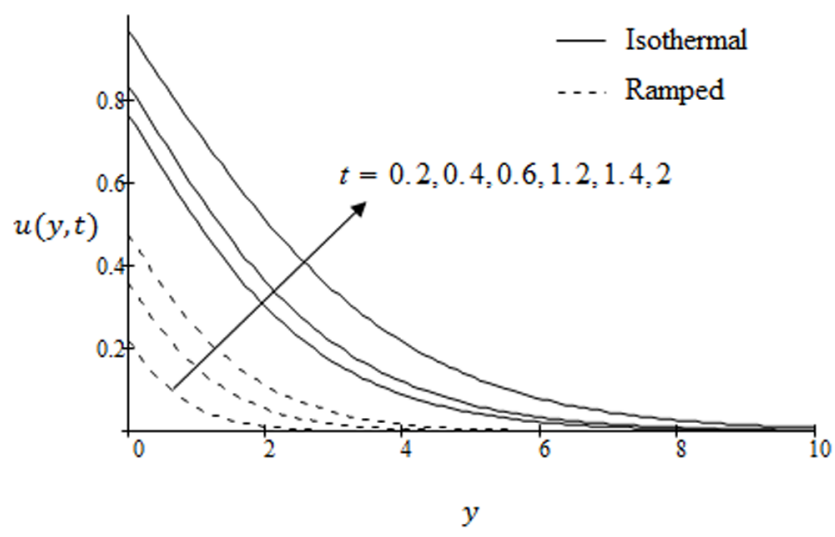

Figure 6. Velocity profiles for different values of $t$ when the plate applies a constant shear stress $f=-0.25$. doi:10.1371/journal.pone.0090280.g006

\subsection{Solution in the absence of magnetic parameter $(M \rightarrow 0)$}

As it is clear from Eqs. (22) and (26) that the temperature and concentration distributions are not effected by the magnetic parameter $M$, and the velocities with $M=0$ for both ramped and constant wall temperature are given by

$$
\begin{aligned}
& u(y, t)=a_{1} \int_{0}^{t}\left(\frac{e^{a_{10}(t-s)} \operatorname{erf}\left(\sqrt{a_{10}(t-s)}\right)}{\left(a_{10}\right)^{\frac{3}{2}}}-\frac{2 \sqrt{t-s}}{\sqrt{\pi} a_{10}}\right) \frac{e^{-K p s-\frac{y^{2}}{4 s}}}{\sqrt{\pi s}} d s \\
& +\left[\frac{a_{1}}{\pi a_{10}} \int_{0}^{t-1} \frac{2 \sqrt{t-1-s} e^{-K p s-\frac{y^{2}}{4 s}}}{\sqrt{s}} d s\right] H(t-1)
\end{aligned}
$$$$
-\left[\frac{a_{1}}{\left(a_{10}\right)^{\frac{3}{2}} \sqrt{\pi}} \int_{0}^{t-1} \frac{\operatorname{erf}\left(\sqrt{a_{10}(t-1-s)}\right) e^{a_{10}(t-1-s)-K_{p} s-\frac{y^{2}}{4 s}}}{\sqrt{s}} d s\right] H(t-1)
$$$$
+a_{4} \int_{0}^{t}\left(\frac{e^{a_{11}(t-s)} \operatorname{erf}\left(\sqrt{a_{11}(t-s)}\right)}{\sqrt{a_{11}}}-\frac{2 \sqrt{t-s}}{\sqrt{\pi} a_{10}}\right) \frac{e^{-K_{p} s-\frac{y^{2}}{4 s}}}{\sqrt{\pi s}} d s
$$$$
+\frac{a_{3}}{a_{10}}\left(t+\frac{\operatorname{Pr}_{\text {eff }} y^{2}}{2}\right) \operatorname{erf} c\left(\frac{y \sqrt{\operatorname{Pr}_{e f f}}}{2 \sqrt{t}}\right)
$$$$
-\frac{a_{3}}{a_{10}} \frac{y \sqrt{\operatorname{Pr}_{e f f}} \sqrt{t}}{\sqrt{\pi}} e^{\frac{-y^{2} \operatorname{Pr}_{e f f}}{4 t}}+\frac{a_{3}}{a_{10}^{2}} \operatorname{erf} c\left(\frac{y \sqrt{\operatorname{Pr}_{e f f}}}{2 \sqrt{t}}\right)
$$$$
-\frac{1}{\sqrt{\pi}} \int_{0}^{t} \frac{f(t-s) e^{-K_{p} s-\frac{y^{2}}{4 s}}}{\sqrt{s}} d s
$$$$
-\frac{a_{3} e^{a_{10} t+y \sqrt{\mathrm{Pr}_{e f f} a_{10}}}}{2 a_{10}^{2}} \operatorname{erf} c\left(\frac{y \sqrt{\operatorname{Pr}_{\text {eff }}}}{2 \sqrt{t}}+\sqrt{a_{10} t}\right)
$$

$$
-\frac{a_{3} e^{a_{10} t-y \sqrt{\operatorname{Pr}_{e f f} a_{10}}}}{2 a_{10}^{2}} \operatorname{erf} c\left(\frac{y \sqrt{\operatorname{Pr}_{e f f}}}{2 \sqrt{t}}-\sqrt{a_{10} t}\right)
$$$$
-\frac{a_{3}}{a_{10}}\left((t-1)+\frac{\operatorname{Pr}_{e f f} y^{2}}{2}\right) \operatorname{erf} c\left(\frac{y \sqrt{\operatorname{Pr}_{e f f}}}{2 \sqrt{t-1}}\right) H(t-1)
$$$$
+\frac{a_{3}}{a_{10}} \frac{y \sqrt{\operatorname{Pr}_{e f f}} \sqrt{t-1}}{\sqrt{\pi}} e^{\frac{-y^{2} \operatorname{Pr}_{e f f}}{4(t-1)}} H(t-1)
$$$$
-\frac{a_{6} e^{a_{11} t-y \sqrt{a_{11} S c}}}{2 a_{11}} \operatorname{erf} c\left(\frac{y \sqrt{S c}}{2 \sqrt{t}}-\sqrt{a_{11} t}\right)
$$$$
-\frac{a_{3}}{a_{10}^{2}} \operatorname{erf} c\left(\frac{y \sqrt{\operatorname{Pr}_{e f f}}}{2 \sqrt{t-1}}\right) H(t-1)+\frac{a_{6}}{a_{11}} \operatorname{erf} c\left(\frac{y \sqrt{S c}}{2 \sqrt{t}}\right)
$$

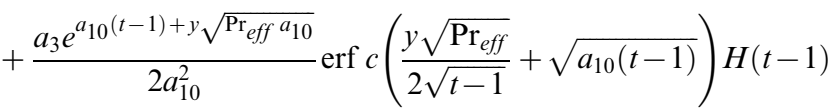

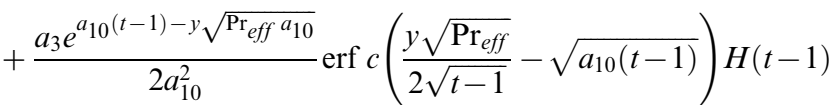$$
-\frac{a_{6} e^{a_{11} t+y \sqrt{a_{11} S c}}}{2 a_{11}} \operatorname{erf} c\left(\frac{y \sqrt{S c}}{2 \sqrt{t}}+\sqrt{a_{11} t}\right),
$$ 


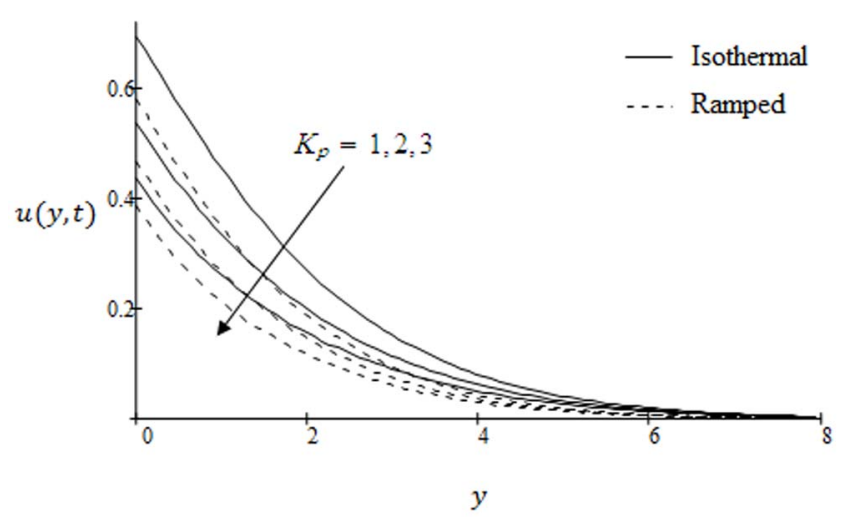

Figure 7. Velocity profiles for different values of $K_{p}$ when the plate applies a constant shear stress $\boldsymbol{f}=-\mathbf{0 . 2 5}$. doi:10.1371/journal.pone.0090280.g007

$$
\begin{aligned}
& u(y, t)=\frac{a_{1}}{\sqrt{\pi a_{10}}} \int_{0}^{t} \frac{e^{a_{10}(t-s)-K p s-\frac{y^{2}}{4 s}} \operatorname{erf}\left(\sqrt{a_{10}(t-s)}\right)}{\sqrt{s}} d s \\
& +\frac{a_{4}}{\sqrt{\pi a_{11}}} \int_{0}^{t} \frac{e^{a_{11}(t-s)-K_{p} s-\frac{y^{2}}{4 s}} \operatorname{erf}\left(\sqrt{a_{11}(t-s)}\right)}{\sqrt{s}} d s
\end{aligned}
$$

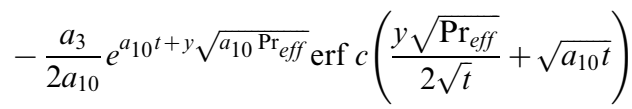

$$
\begin{aligned}
& -\frac{a_{3}}{2 a_{10}} e^{a_{10} t-y \sqrt{a_{10} \mathrm{Pr}_{e f f}}} \operatorname{erf} c\left(\frac{y \sqrt{\operatorname{Pr}_{e f f}}}{2 \sqrt{t}}-\sqrt{a_{10} t}\right) \\
& +\frac{a_{3}}{a_{10}} \operatorname{erf} c\left(\frac{y \sqrt{\operatorname{Pr}_{e f f}}}{2 \sqrt{t}}\right)+\frac{a_{6}}{a_{11}} \operatorname{erf} c\left(\frac{y \sqrt{S c}}{2 \sqrt{t}}\right) \\
& -\frac{a_{6}}{2 a_{11}} e^{a_{11} t+y \sqrt{a_{11} S c}} \operatorname{erf} c\left(\frac{y \sqrt{S c}}{2 \sqrt{t}}+\sqrt{a_{11} t}\right) \\
& -\frac{1}{\sqrt{\pi}} \int_{0}^{t} \frac{f(t-s) e^{-K_{p} s-\frac{y^{2}}{4 s}}}{\sqrt{s}} d s \\
& -\frac{a_{6}}{2 a_{11}} e^{a_{11} t-y \sqrt{a_{11} S c}} \operatorname{erf} c\left(\frac{y \sqrt{S c}}{2 \sqrt{t}}-\sqrt{a_{11} t}\right) .
\end{aligned}
$$

\section{Special Cases}

As we noted that the solutions for velocity obtained in Section 3, are more general. Therefore, we want to discuss some special cases of the present solutions together with some limiting solutions in order to know more about the physical insight of the problem. Hence, we discuss the following important special cases in the case of ramped wall temperature whose technical relevance is wellknown in the literature. Similarly we can discuss some special cases of constant wall temperature solutions.

\subsection{Case-I: $f(t)=f H(t)$}

In this first case we take the arbitrary function $f(t)=f H(t)$, where $f$ is a dimensionless constant and $H(\cdot)$ denotes the unit step function. After time $t=0$, the infinite vertical plate applies a constant shear stress to the fluid. The convective part of the velocity remains unchanged while the mechanical part takes the following form

$$
u_{m}(y, t)=-\frac{f}{\sqrt{\pi}} \int_{0}^{t} \frac{e^{-\frac{y^{2}}{4 s}-H_{1} s}}{\sqrt{s}} d s
$$

equivalently

$$
u_{m}(y, t)=-\frac{f}{\sqrt{H_{1}}} e^{-y \sqrt{H_{1}}}+\frac{2 f}{\sqrt{\pi}} \int_{\sqrt{t}}^{\infty} e^{-\frac{y^{2}}{4 z^{2}}-H_{1} z^{2}} d z
$$

for $K_{p} \neq 0, M \neq 0$. Moreover, if we take $M=0$, Eq. (50) reduces to the form

$$
u_{m}(y, t)=-\frac{f}{\sqrt{K_{p}}} e^{-y \sqrt{K_{p}}}+\frac{2 f}{\sqrt{\pi}} \int_{\sqrt{t}}^{\infty} e^{-\frac{y^{2}}{4 z^{2}}-K_{p} z^{2}} d z
$$

which is equivalent to [28]; Eq. (28) with the correction of $\sqrt{K_{p}}$.

Furthermore, in the absence of both $K_{p}=0$ and $M=0$, Eq. (50) is identical with [27]; Eq. (23)

$$
u_{m}(y, t)=-\frac{f}{\sqrt{\pi}} \int_{0}^{t} \frac{e^{-\frac{y^{2}}{4 s}}}{\sqrt{s}} d s
$$

\subsection{Case-II: $f(t)=f \sin (\omega t)$}

In the second case, we take the arbitrary function of the form $f(t)=f \sin (\omega t)$ in which the plate applies an oscillating shear stress to the fluid. Here $\omega$ denotes the dimensionless frequency of the shear stress. As previously, the convective part of velocity remains the same whereas the mechanical part takes the form

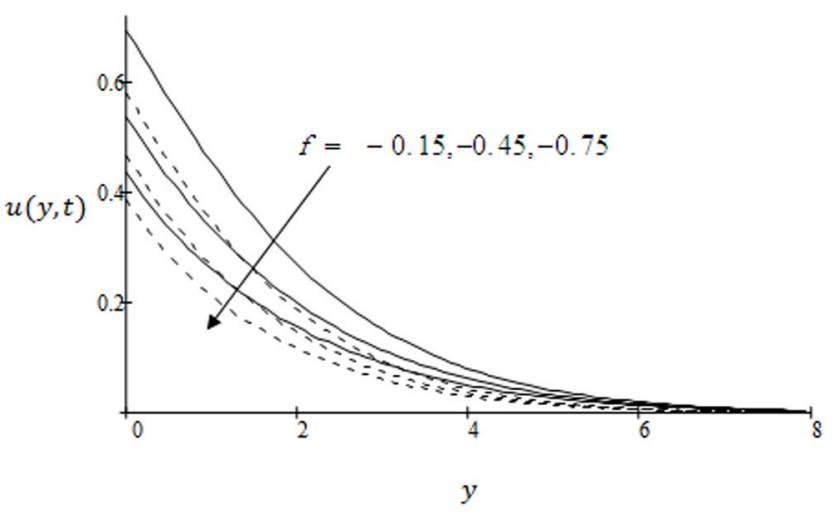

Figure 8. Velocity profiles for different values of constant shear stress $f$.

doi:10.1371/journal.pone.0090280.g008 


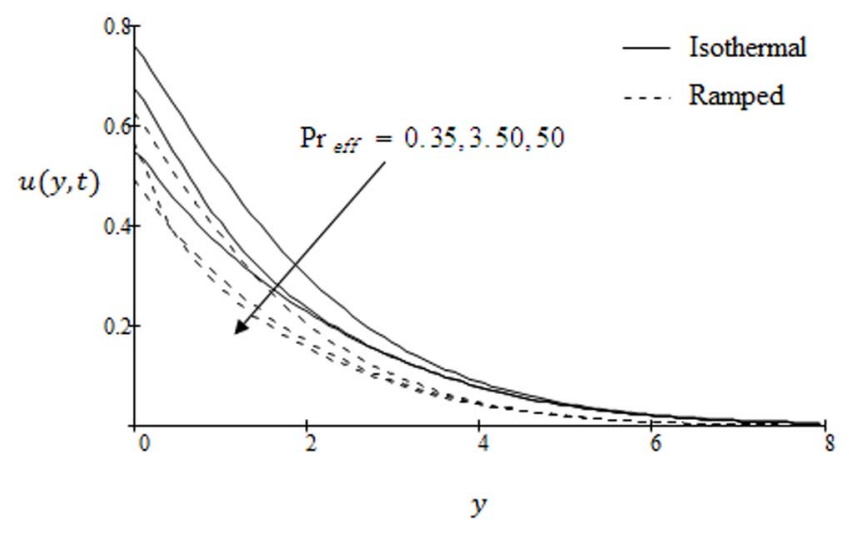

Figure 9. Velocity profiles for different values of $\operatorname{Pr}_{\text {eff }}$ when the plate applies a constant shear stress $f=-0.25$.

doi:10.1371/journal.pone.0090280.g009

$$
u_{m}(y, t)=-\frac{f}{\sqrt{\pi}} \int_{0}^{t} \frac{\sin (\omega t-\omega s) e^{-\frac{y^{2}}{4 s}-H_{1} s}}{\sqrt{s}} d s .
$$

It can be further written as a sum of the steady-state and transient solutions

$$
u_{m}(y, t)=u_{m s}(y, t)+u_{m t}(y, t)
$$

where

$$
\begin{aligned}
& u_{m s}(y, t)=-\frac{f}{\sqrt{\pi}} \int_{0}^{t} \frac{\sin (\omega t-\omega s) e^{-\frac{y^{2}}{4 s}-H_{1} s}}{\sqrt{s}} d s, \\
& u_{m t}(y, t)=\frac{f}{\sqrt{\pi}} \int_{t}^{\infty} \frac{\sin (\omega t-\omega s) e^{-\frac{y^{2}}{4 s}-H_{1} s}}{\sqrt{s}} d s .
\end{aligned}
$$

By taking $M=0$, the steady-state component reduces to [28]; Eq. (35)

$$
u_{m s}(y, t)=-\frac{f}{\sqrt{\pi}} \int_{0}^{t} \frac{\sin (\omega t-\omega s) e^{-\frac{y^{2}}{4 s}-K_{p} s}}{\sqrt{s}} d s
$$

In addition when $K_{p}=0$, physically it corresponds to the absence of porous effects and Eq. (58) results in

$$
u_{m s}(y, t)=-\frac{f}{\sqrt{\pi}} \int_{0}^{t} \frac{\sin (\omega t-\omega s) e^{-\frac{y^{2}}{4 s}}}{\sqrt{s}} d s,
$$

which can be written in simplified form as

$$
u_{m s}(y, t)=\frac{f}{\sqrt{\omega}} \exp \left(-y \sqrt{\frac{\omega}{2}}\right) \cos \left(\omega t-y \sqrt{\frac{\omega}{2}}+\frac{\pi}{4}\right),
$$

equivalent to [27]; Eq. (33).

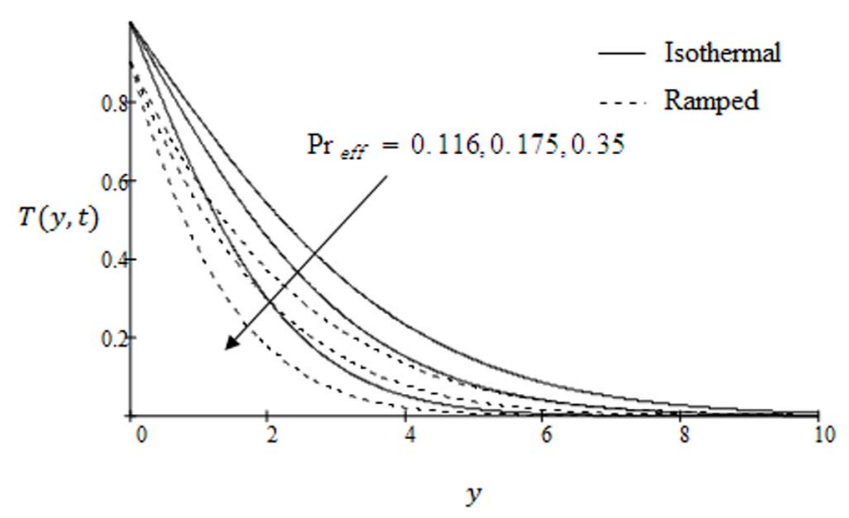

Figure 10. Temperature profile for different values of $\operatorname{Pr}_{\text {eff }}$. doi:10.1371/journal.pone.0090280.g010

\subsection{Case-III: $f(t)=f t^{a}(a>0)$}

In the final case, we take $f(t)=f t^{a}$, in which the plate applies an accelerating shear stress to the fluid where the mechanical part takes the following form

$$
u_{m}(y, t)=-\frac{f}{\sqrt{\pi}} \int_{0}^{t} \frac{(t-s)^{a} e^{-\frac{y^{2}}{4 s}-H_{1} s}}{\sqrt{s}} d s .
$$

The corresponding solution for $M=0$, namely

$$
u_{m}(y, t)=-\frac{f}{\sqrt{\pi}} \int_{0}^{t} \frac{(t-s)^{a} e^{-\frac{y^{2}}{4 s}-K_{p} s}}{\sqrt{s}} d s,
$$

is identical with [28]; Eq. (32).

Additionally, if we take $K_{p}=0$, Eq. (62) yields

$$
u_{m}(y, t)=-\frac{f}{\sqrt{\pi}} \int_{0}^{t} \frac{(t-s)^{a} e^{-\frac{y^{2}}{4 s}}}{\sqrt{s}} d s
$$

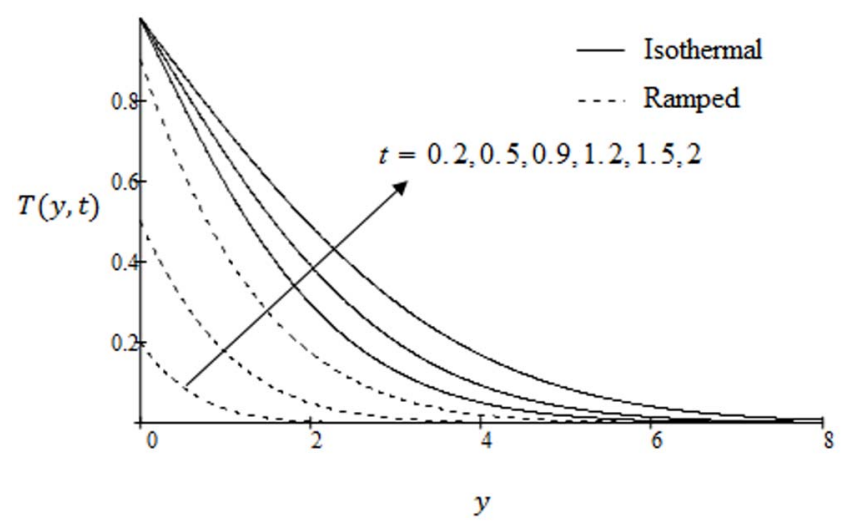

Figure 11. Temperature profiles for different values of $\boldsymbol{t}$. doi:10.1371/journal.pone.0090280.g011 


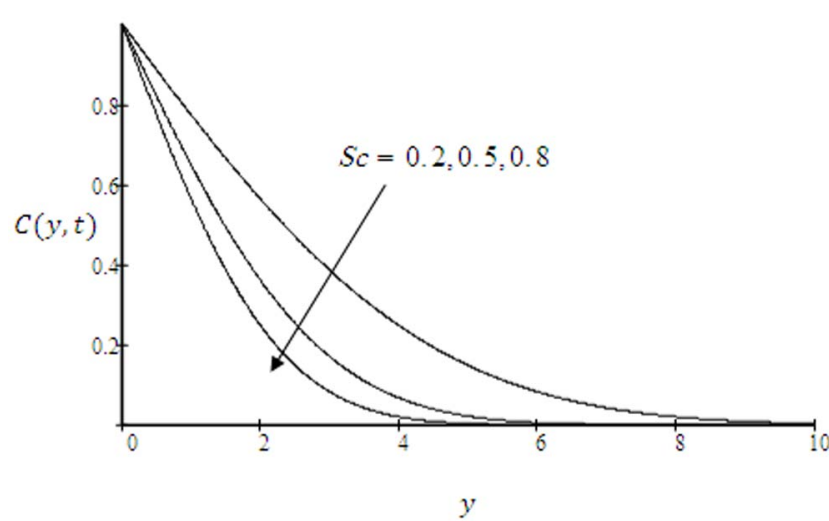

Figure 12. Concentration profiles for $t=1.2$ and different values of $S c$.

doi:10.1371/journal.pone.0090280.g012

\section{Results and Discussion}

In order to understand the physical aspects of the problem, the numerical results for velocity, temperature and concentration are computed and plotted for various parameters of interest such as magnetic parameter $M$, porosity parameter $K_{p}$, effective Prandtl number $\operatorname{Pr}_{\text {eff }}$, Grashof number $G r$, modified Grashof number $G m$, dimensionless time $t$, Schmidt number $S c$ and shear stress $f$. The graphs for velocity are shown in Figs. 2-9 where $t=1.2$ corresponds to isothermal velocity and $t=0.9$ is for ramped velocity. Figs. 10 and 11 are plotted to show the temperature variations for two types of boundary conditions namely ramped and constant wall temperatures. Furthermore, Figs. 12 and 13 are displayed to show variations in fluid concentration. Fig. 2 illustrate the influence of Grashof number $G r$ on the velocity. It is observed that velocity increases with increasing $G r$. This implies that thermal buoyancy force tends to accelerate velocity for both ramped temperature and isothermal plates. In Fig. 3 the velocity profiles for different values modified Grashof number $\mathrm{Gm}$ are shown. It is found that velocity increases on increasing $G m$ for both ramped temperature and isothermal plate. Further, it can be observed that the velocity and boundary layer thickness decrease along $y$ with increasing distance from the the leading edge. Moreover, we observed that the amplitude of velocity in case of isothermal plate is greater and converges slowly as compare to ramped velocity. In Fig. 4 the velocity profiles are shown for different values of Schmidt number Sc. It is observed that the velocity decreases with increasing Schmidt number. The velocity profiles for different values of magnetic parameter $M$ are shown in Fig. 5. The range of magnetic field is taken from 0 to 2 . It is found that the velocity is decreasing with increasing values of $M$ in both cases of ramped and isothermal plates. Physically, it is true due to the fact that increasing values of $M$ causes the frictional force to increase which tends to resist the fluid flow and thus reducing its velocity. It is further observed that when the magnetic field imposed on the flow is zero $(M=0)$, the MHD effect vanishes and the flow is termed as hydrodynamic flow.

Fig. 6 are plotted to see the difference between the ramped and isothermal plate velocities. The values of $t<1$ correspond to ramp velocity whereas $t>1$ is for isothermal plate. It is found that ramp velocity is less than isothermal plate and converges faster. Further velocity in both cases increases with increasing time. The effects of inverse permeability parameter $K_{p}$ on the velocity profiles are presented in Fig. 7. It is found that velocity decreases with increasing $K_{p}$ in both cases of ramp and isothermal plate.

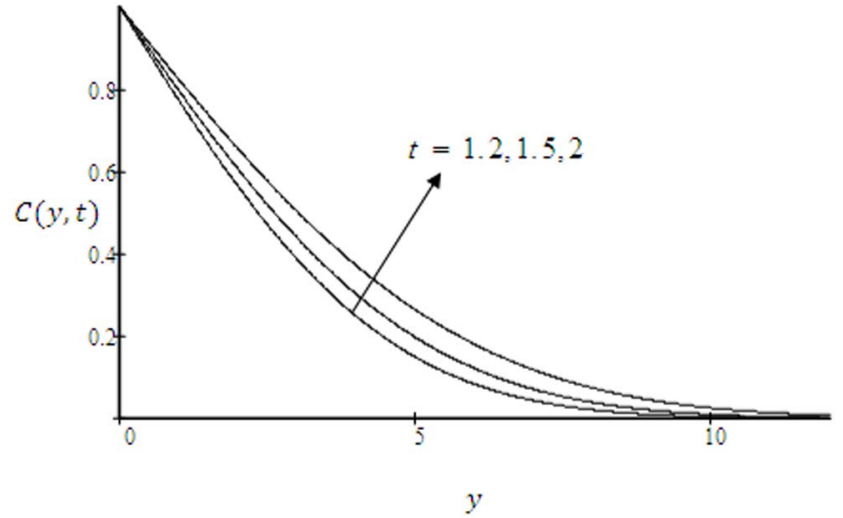

Figure 13. Concentration profiles for $S c=0.2$ and different values of $t$.

doi:10.1371/journal.pone.0090280.g013

Physically, it is due to the fact that increasing permeability of the porous medium increases the resistance and consequently velocity decreases. This observation is an excellent agreement with the previous study [28]; Fig. 3. The effects of the shear stress $f$ induced by the bounding plate on the non-dimensional velocity profiles are shown in Fig. 8. The velocity of fluid is found to decrease with increasing $f$ in both cases of ramped velocity and isothermal plate. Graphical results to show the influence of the effective Prandtl number $\operatorname{Pr}_{\text {eff }}$ on velocity profiles are presented in Fig. 9. It is observed that the velocity is a decreasing function with respect to $\operatorname{Pr}_{\text {eff }}$. These graphical results are in accordance with [28]; Fig. 2.

The temperature variations against $y$ for various values of effective Prandtl number are highlighted in Fig. 10. The significant decrease of the temperature is found as a result of an increase of the effective Prandtl number. The fluid temperature decreases from maximum at the boundary to a minimum value as far from the plate in both cases of ramped and constant temperature. In Fig. 11 we have shown the temperature variations for two types of boundary conditions ramped and constant plate temperatures. It is noted that the fluid temperature is greater in the case of isothermal plate than in the case of ramped temperature at the plate. This should be expected since in the latter case, the heating of the fluid takes place more gradually than in the isothermal case [18]. Moreover, with increasing time, the temperature is found to increase in both cases of ramped and constant wall temperature. The concentration profiles for different values of Schmidt number $S c$, are shown in Fig. 12. It is clear from this figure that the concentration profiles and the concentration boundary layer thickness decrease with increasing values of $S c$. Physically, it is true, since increase of $S c$ means decrease of molecular diffusivity which results in a decrease of concentration boundary layer. The concentration profiles for different values of time $t$ are presented in Fig. 13. It is observed that concentration profiles increase with increasing $t$.

\section{Conclusions}

The purpose of this work was to analyze the unsteady MHD free convection flow of an incompressible viscous fluid over an infinite plate with ramped wall temperature and applies an arbitrary shear stress to the fluid. Exact solutions for velocity, temperature (for both cases of ramped and constant wall temperature) and concentration are obtained using the Laplace transform technique and expressed in terms of the complementary error function. They satisfy all imposed initial and boundary 
conditions. These solutions are plotted in various figures for different parameters of interest. It is found that velocity of the fluid $u(y, t)$ can be written as a sum of its mechanical and thermal components $u_{m}(y, t)$, respectively $u_{t}(y, t)$. For the velocity solution in which the plate applies an oscillating shear stress to the fluid $f(t)=f \sin (\omega t)$, the mechanical part can be further written as a sum of the steady-state and transient solutions $u_{m s}(y, t)$, respectively $u_{m t}(y, t)$. The thermal boundary layer thickness in case of ramped wall temperature is less than isothermal wall temperature. Magnetic parameter $M$ retards whereas the inverse permeability parameter $K_{p}$ enhances the fluid motion. The thermal boundary

\section{References}

1. Khan I, Ali F, Sharidan S, Norzieha M (2011) Effects of Hall current and mass transfer on the unsteady magnetohydrodynamic flow in a porous channel. J Phys Soc Jpn 80: 064401.

2. Das K, Jana S (2010) Heat and mass transfer effects on unsteady MHD free convection flow near a moving vertical plate in porous medium. Bull Soc Math Banja Luka 17: 15-32.

3. Das SS, Satapathy A, Das JK, Panda JP (2009) Mass transfer effects on MHD flow and heat transfer past a vertical porous plate through a porous medium under oscillatory suction and heat source. Int J Heat Mass Transfer 52: 5962 5969.

4. Chandrakala P (2011) Radiation effects on flow past an impulsively started vertical oscillating plate with uniform heat flux. Int J Dyn Fluids 7: 1-8.

5. Das SS, Parija S, Padhy RK, Sahu M (2012) Natural convection unsteady magneto-hydrodynamic mass transfer flow past an infinite vertical porous plate in presence of suction and heat sink. Int J Energy Environ 3: 209-222.

6. Das SS, Maity M, Das JK (2012) Unsteady hydromagnetic convective flow past an infinite vertical porous flat plate in a porous medium, Int J Energy Environ 3: 109-118.

7. Narahari M, Ishaq A (2011) Radiation effects on free convection flow near a moving vertical plate with Newtonian heatin. J Appl Sci 11: 1096-1104.

8. Narahari M, Nayan MY (2011) Free convection flow past an impulsively started infinite vertical plate with Newtonian heating in the presence of thermal radiation and mass diffusion. Turkish J Eng Env Sci 35: 187-198.

9. Hussanan A, Khan I, Sharidan S (2013) An exact analysis of heat and mass transfer past a vertical plate with Newtonian heating. J Appl Math Article ID 434571 http://dx.doi.org/10.1155/2013/434571.

10. Das SS, Biswal SR, Tripathy UK, Das P (2011) Mass transfer effects on unsteady hydromagnetic convective flow past a vertical porous plate in a porous medium with heat source. J Appl Fluid Mech 4: 91-100.

11. Toki CJ, Tokis JN (2007) Exact solutions for the unsteady free convection flows on a porous plate with time-dependent heating. ZAMM Z Angew Math Mech 87: $4-13$.

12. Senapati N, Dhal RK, Das TK (2012) Effects of chemical reaction on free convection MHD flow through porous medium bounded by vertical surface with slip flow region. Amer J Comput Appl Math 2: 124-135.

13. Khan I, Fakhar K, Sharidan S (2011) Magnetohydrodynamic free convection flow past an oscillating plate embedded in a porous medium. J Phys Soc Jpn 80: 104401 .

14. Shercliff JA (1965) Textbook of magnetohydrodynamics. London: pergamon press.

15. Hayat T, Khan I, Ellahi R, Fetecau G (2008) Some MHD flows of a second grade fluid through the porous medium. J Porous Media 11: 389-400.

16. Jha BK, Apere CA (2010) Combined effect of hall and ion-slip currents on unsteady mhd couette flows in a rotating system. J Phys Soc Jpn 79:104401.

17. Fetecau C, Vieru D, Corina Fetecau, Akhter S (2013) General solutions for magnetohy-drodynamic natural convection flow with radiative heat transfer and layer, as well as the temperature of the fluid, increases in time and decreases with respect to the effective Prandtl number $\operatorname{Pr}_{\text {eff }}$. The concentration boundary layer thickness decreases with increasing values of $S c$ whereas increases with increasing $t$.

\section{Author Contributions}

Conceived and designed the experiments: IK. Performed the experiments: AK. Analyzed the data: Su. Contributed reagents/materials/analysis tools: SS. Wrote the paper: FA.

slip condition over a moving plat. Z Naturforsch 68a 659-667 / DOI: 10.5560/ ZNA.2013-0041.

18. Chandran P, Sacheti NC, Singh AK (2005) Natural convection near a vertical plate with ramped wall temperature. Heat Mass Transf 41: 459-464.

19. Narahari M, Beg OA, Ghosh SK (2011) Mathematical modelling of mass transfer and free convection current effects on unsteady viscous flow with ramped wall temperature. World J Mech 1: 176-184.

20. Rajesh V (2011) Chemical reaction and radiation effects on the transient MHD free convection flow of dissipative fluid past an inflnite vertical porous plate with ramped wall temperature. Chem Ind Chem Eng Quar 17: 189-198.

21. Patral RR, Das S, Jana RN, Ghosh SK (2012) Transient approach to radiative heat transfer free convection flow with ramped wall temperature. J Appl Fluid Mech: 59-13.

22. Seth GS, Mahatoo GK, Sarkar S (2013) Effects of Hall current and rotation on MHD natural convection flow past an impulsively moving vertical plate with ramped temper-ature in the presence of thermal diffusion with heat absorption. Int J Energy Tech 5: 1-12.

23. Samiulhaq, Khan I, Ali F, Sharidan S (2012) MHD free convection flow in a porous medium with thermal diffusion and ramped wall temperature. J Phys Soc Jpn 81: 044401.

24. Ghara N, Das S, Maji SL, Jana RN (2012) Effect of radiation on MHD free convection flow past an impulsively moving vertical plate with ramped wall temperature. Am J Sci Ind Res 3: 376-386.

25. Das S, Mandal C, Jana RN (2012) Effects of radiation on unsteady Couette flow between two vertical parallel plates with ramped wall temperature. Int J Comput Appl 39: 37-45.

26. Navier CLMH (1827) Sur les lois dee mouvement des fluids. Mem Acad R Sci Inst Fr 6: 389-440.

27. Fetecau C, Corina Fetecau, Rana M (2011) General solutions for the unsteady flow of second-grade fluids over an inflnite plate that applies arbitrary shear to the fluid. Z Naturfors Sect A-J Phys Sci 66: 753-759.

28. Corina Fetecau, Rana M, Fetecau C (2013) Radiative and porous effects on free con-vection flow near a vertical plate that applies shear stress to the fluid. Z Naturfors Sect A-J Phys Sci 68: 130-138.

29. Magyari E, Pantokratoras A (2011) Note on the effect of thermal radiation in the linearized Rosseland approximation on the heat transfer characteristics of various boundary layer flows. Int Commun Heat Mass Transf 38: 554-556.

30. Narahari M, Dutta BK (2012) Effects of thermal radiation and mass diffusion on free convection flow near a vertical plate with Newtonian heating. Chem Eng Commun 199: 628-643.

31. Ghosh SK, Beg OA (2008) Theoretical analysis of radiative effects on transient free convection heat transfer past a hot vertical surface in porous media. Nonlin Anal Model Control 13: 419-432.

32. Seethamahalakshmi, Ramana Reddy GV, Prasad BDCN (2011) Unsteady MHD free convection flow and mass transfer near a moving vertical plate in the presence of thermal radiation. Adv Appl Sc Res 2: 261-269. 